\title{
Current approaches to the diagnosis and treatment of idiopathic pulmonary fibrosis in Europe: the AIR survey
}

\author{
Vincent Cottin ${ }^{1,2}$
}

\begin{abstract}
Affiliations: ${ }^{1}$ Hospices Civils de Lyon, Hôpital Louis Pradel, Service de pneumologie - Centre de référence national des maladies pulmonaires rares, Lyon, France. ${ }^{2}$ Université de Lyon, Université Claude Bernard Lyon 1, INRA, UMR754 IFR 128, Lyon, France.
\end{abstract}

Correspondence: Vincent Cottin, Hôpital Louis Pradel, Claude Bernard Lyon 1 University, 28 Avenue du Doyen Lepine, 69677 Lyon, France. E-mail: vincent.cottinachu-lyon.fr

ABSTRACT This review presents the results of the 2013 Advancing IPF Research (AIR) survey, which assessed current approaches to the diagnosis and treatment of idiopathic pulmonary fibrosis (IPF) by experienced physicians. A total of 149 physicians, predominantly from European countries, replied to the 28-question survey. The results of the AIR survey were compared with a similar survey of 509 French pulmonologists conducted by the French National Reference Centre and the Network of Regional Competence Centres for Rare Lung Diseases.

A number of positive findings emerged from the AIR survey, including the high level of multidisciplinary team involvement in both diagnosis and management. This survey, when taken together with the French survey, suggests that there is still a need to improve earlier diagnosis of IPF.

@ERSpublications

Survey on IPF in Europe reveals need to improve early diagnosis and management http://ow.ly/vnKvo

\section{Introduction}

Idiopathic pulmonary fibrosis (IPF) is a chronic respiratory disease associated with a progressive decline in lung function, which occurs as a result of fibrosis of the lung parenchyma $[1,2]$. The clinical course of IPF is largely unpredictable and, despite extensive research, the precise molecular and cellular mechanisms underlying its pathogenesis remain to be elucidated [1-5]. The classification, nomenclature, diagnosis and treatment of IPF have undergone numerous changes in the past. In addition, the complex nature and highly variable course of IPF among individuals complicates diagnosis and treatment. The 2011 American Thoracic Society/European Respiratory Society/Japanese Respiratory Society/Latin American Thoracic Society (ATS/ERS/JRS/ALAT) guidelines called for a multidisciplinary team (MDT) approach for the diagnosis of IPF whereby information from several sources (e.g. clinical, functional, imaging, biology and, if available, pathology data) is combined and evaluated [6]. In practice, however, it may not always be possible to have access to all the required MDT members. For these reasons, there is likely to be a degree of variation in clinical practice, both in terms of diagnosis and management of IPF, between different centres or countries [7].

In November 2013, a European meeting (Advancing IPF Research (AIR)) of respiratory specialists and experts in IPF was held in Nice, France. A survey of participants was conducted prior to the meeting with

Received: March 102014 | Accepted after revision: April 012014

Conflict of interest: Disclosures can be found alongside the online version of this article at err.ersjournals.com

Provenance: Publication of this peer-reviewed article was sponsored by InterMune International AG, Muttenz, Switzerland (article sponsor, European Respiratory Review issue 132).

Copyright OERS 2014. ERR articles are open access and distributed under the terms of the Creative Commons Attribution Non-Commercial Licence 4.0. 
the objectives of assessing the current diagnostic modalities of IPF, the current management of IPF including pharmacological therapy by physicians experienced in IPF and management of comorbidities. The results of this survey are presented here and are compared with the results of a comparable survey previously conducted in France in 2012 [8].

\section{Survey methodology and description}

The AIR 2013 survey consisted of 28 questions relating to the clinical management of patients with IPF and was distributed online to all participants registering attendance at the AIR meeting. Completion of the survey was required between October 24, 2013 and November 7, 2013. Responses were collected from a total of 149 delegates from 26 countries, predominantly in Europe.

The French survey consisted of a semi-guided questionnaire conducted by telephone or e-mail and involved all French pulmonologists as part of an initiative by the French National Reference Centre and the Network of Regional Competence Centres for Rare Lung Diseases [8]. A total of 2608 French pulmonologists were initially contacted and eventually 509 pulmonologists involved in the management of IPF patients participated. The French survey was conducted from December 7, 2011 to February 18, 2012.

\section{AIR survey results}

\section{Characteristics of IPF patients seen in clinical practice}

The AIR survey responses showed that the mean number of patients that physicians managed annually was 39 (range 3-250 patients). According to the French survey, the mean number of patients managed annually
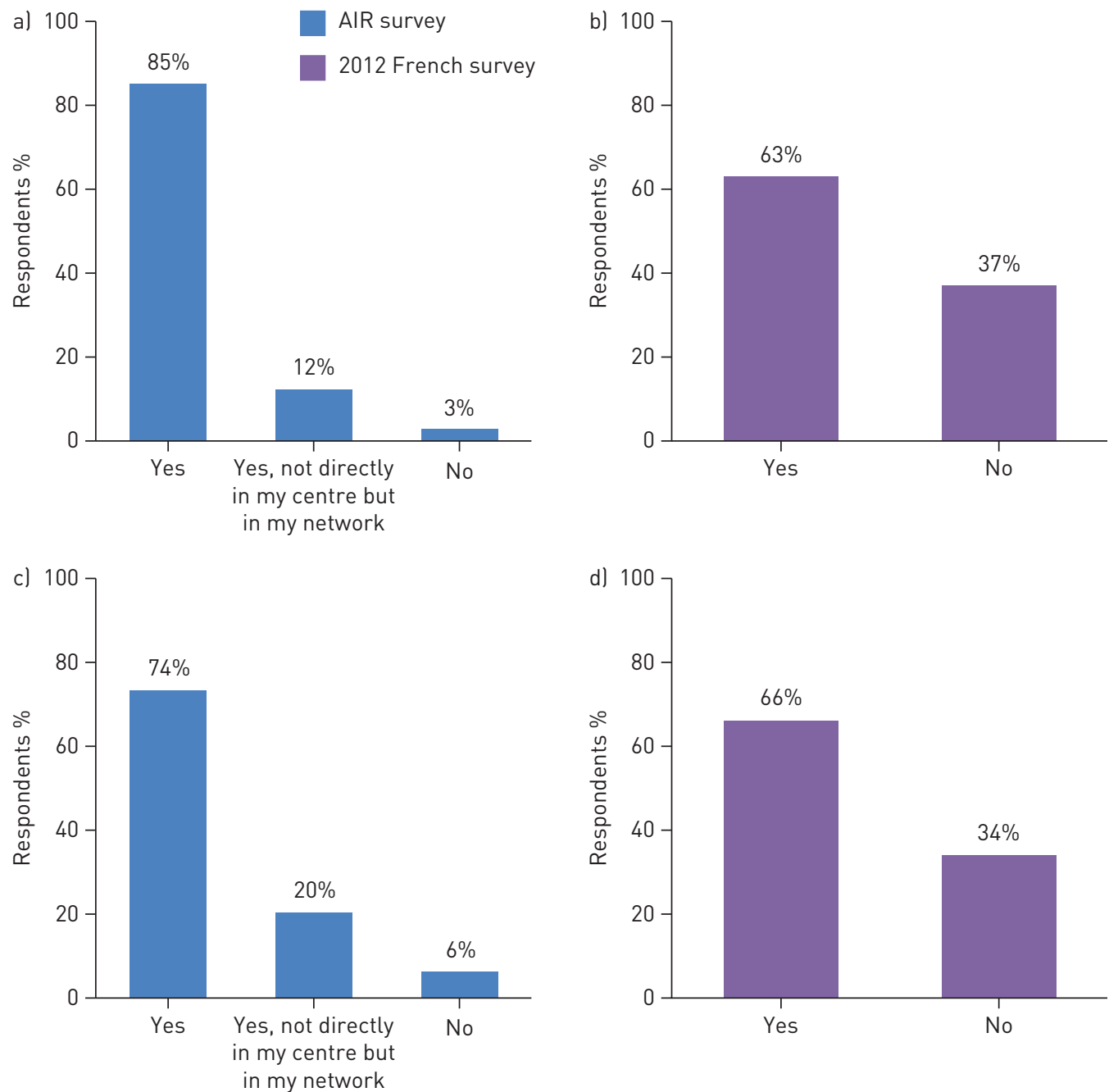

FIGURE 1 Proportion of participants with access to a, b) a radiologist and c, d) a pathologist experienced in interstitial lung diseases to discuss cases of suspected idiopathic pulmonary fibrosis (IPF) in the a, c) 2013 Advancing IPF Research (AIR) survey and b, d) 2012 French survey. In total, there were 147 respondents. 

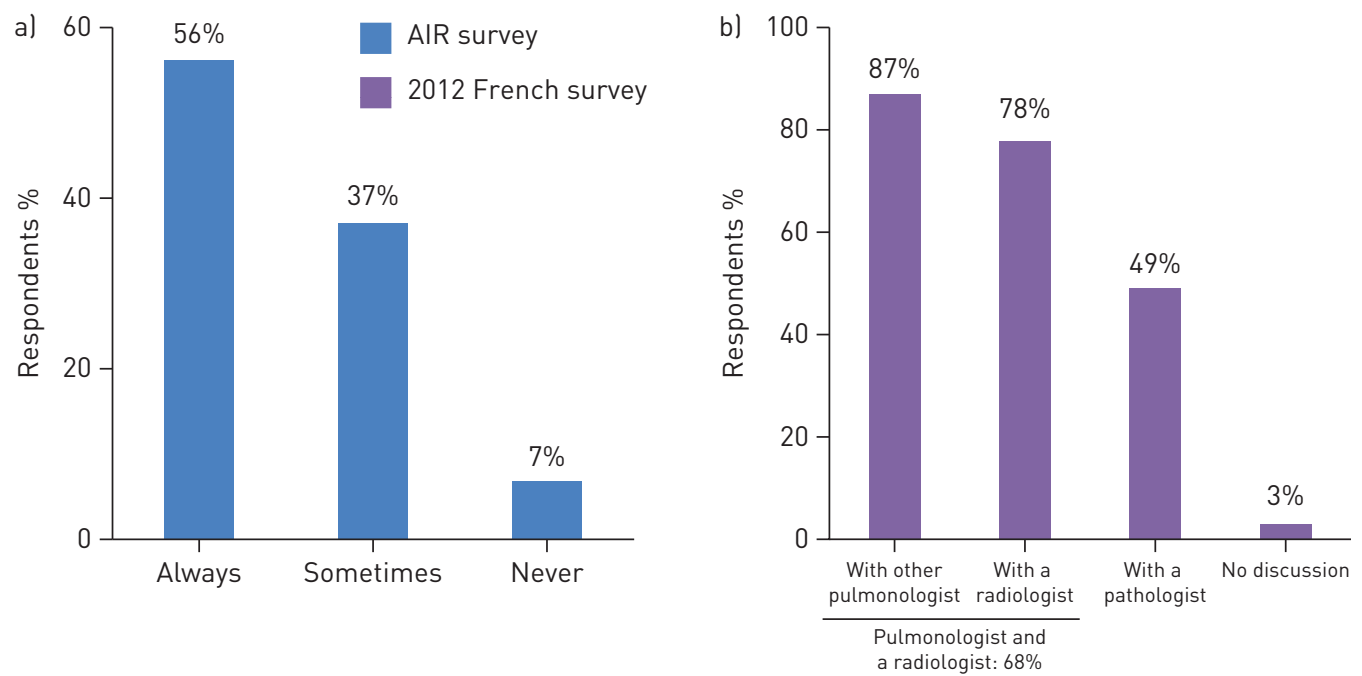

FIGURE 2 Involvement of a multidisciplinary team in diagnosis in a) the 2013 Advancing IPF Research (AIR) survey and b) the 2012 French survey. In total, there were 146 respondents in each survey.

was 56, although this was specific to specialised interstitial lung disease university centres. As might be expected, the majority of patients seen by AIR respondents were elderly (aged 66-80 years) with a history of smoking; the majority of AIR respondents said that $>60 \%$ of their patients had a smoking history. Most of the patients seen by the AIR respondents had mild-to-moderate IPF, characterised by a forced vital capacity (FVC) $>50 \%$ predicted. This compared similarly with the French survey, where the majority of IPF patients $(58 \%)$ also had mild-to-moderate disease (FVC $>50 \%$ predicted and a diffusing capacity of the lung for carbon monoxide $>35 \%$ ) at the time of diagnosis.

\section{Current diagnosis of IPF}

AIR survey respondents and the French survey respondents reported that they had good access to a specialised radiologist to discuss cases of suspected IPF (fig. 1). Similarly, participants in both surveys were able to consult with a specialised pathologist if further information was needed regarding the diagnosis (fig. 1). In both surveys, the vast majority of IPF cases were diagnosed in the context of a MDT. Only 7\% of AIR respondents reported never making the diagnosis of IPF via a MDT and only 3\% of French pulmonologists reported that the diagnosis of IPF was made "without discussion" with other team members (fig. 2).

Based on the responses to the AIR survey, patients were likely to have experienced symptoms for an average duration of 9-12 months prior to diagnosis (fig. 3) and had often seen two or more physicians prior to a confirmed diagnosis of IPF. When AIR respondents were asked what initial symptoms most frequently revealed IPF, dyspnoea on exertion, persistent cough, velcro crackles at lung auscultation and incidental findings on chest computed tomography were all frequently cited (fig. 4). At diagnosis, 94\% of AIR

FIGURE 3 Average duration of symptoms before a diagnosis of idiopathic pulmonary fibrosis (IPF) is confirmed in the 2013 Advancing IPF Research survey. In total, there were 144 respondents.

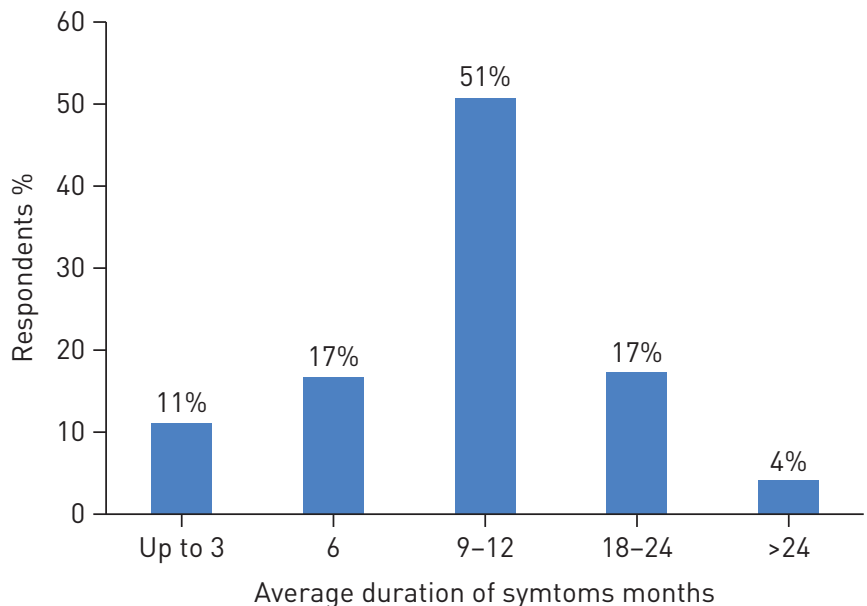




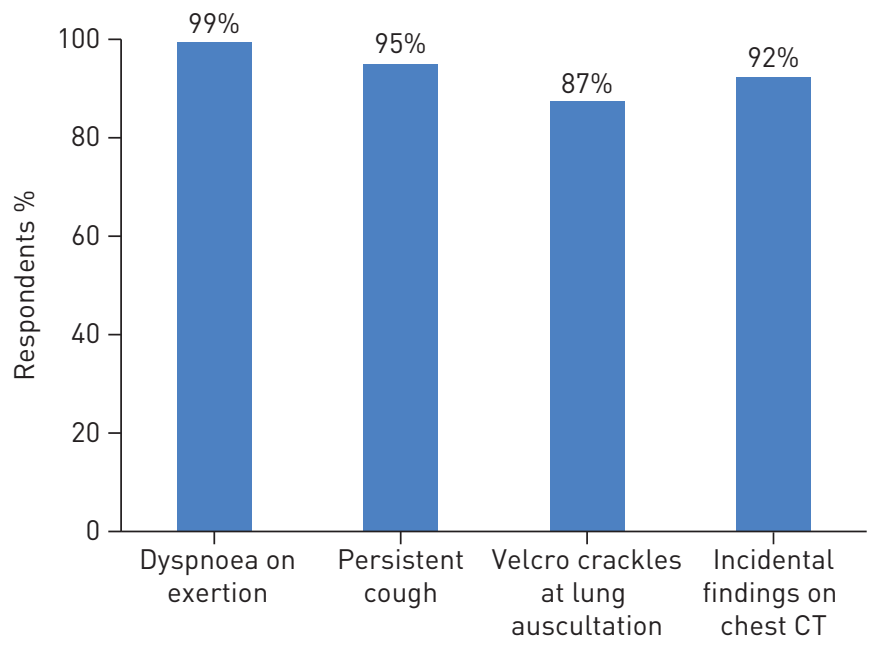

Symptoms
FIGURE 4 Initial symptoms revealing the diagnosis of idiopathic pulmonary fibrosis (IPF) in the 2013 Advancing IPF Research survey and the proportion of physicians who made a diagnosis of IPF in patients referred/who sought medical advice for each of the symptoms. In total, there were 145 respondents. CT: computed tomography.

respondents stated that they had explored the family history of patients but only $29 \%$ performed genetic investigations in the presence of a family history. Although a lower proportion of French survey respondents enquired about family history, a considerably higher number of physicians (77\%) conducted genetic testing if the patient did have a family history.

\section{IPF therapeutic management}

The therapeutic management of patients with mild-to-moderate IPF was most often determined in the context of a MDT or in consultation with other pulmonary specialists. The most commonly initiated

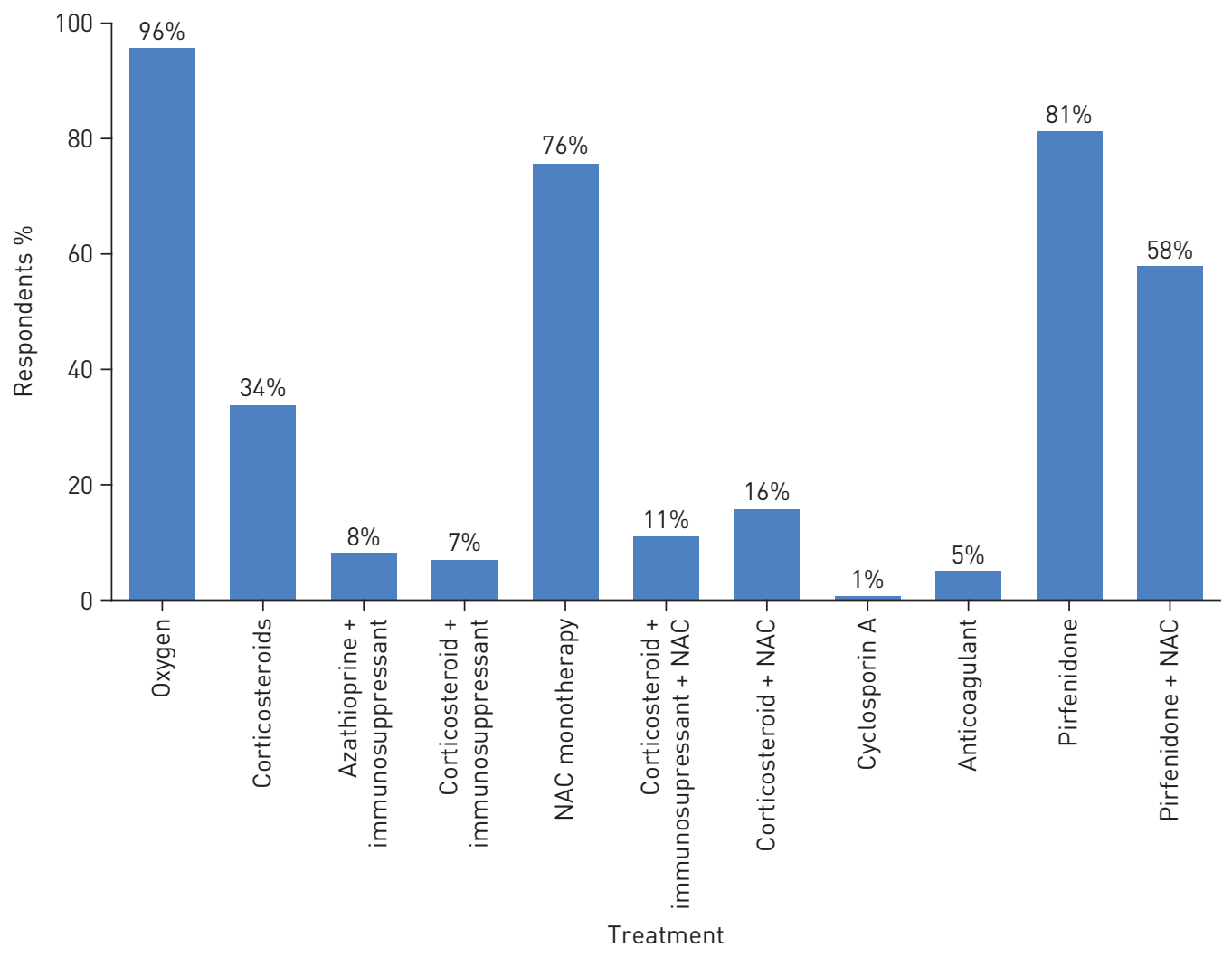

FIGURE 5 Percentage of physicians who prescribed each of the specified treatments in patients diagnosed with definite idiopathic pulmonary fibrosis (IPF) in the 2013 Advancing IPF Research survey. In total, there were 145 respondents. NAC: $N$-acetylcysteine. 


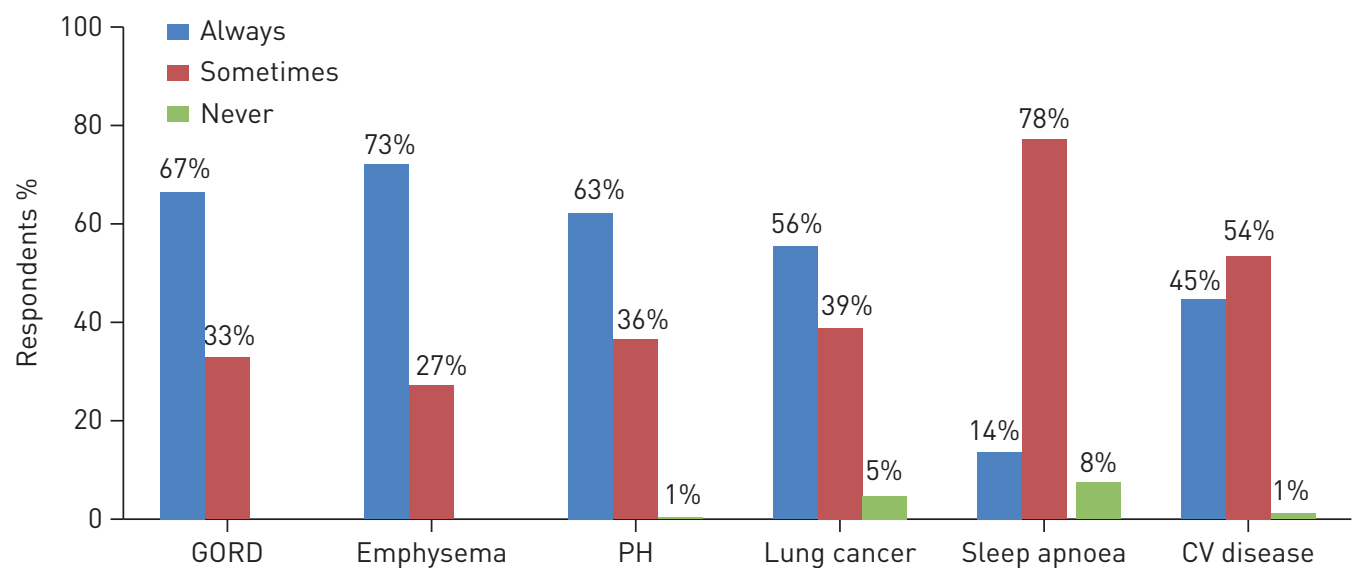

FIGURE 6 Comorbidities/associated conditions/complications assessed in patients diagnosed with idiopathic pulmonary fibrosis (IPF) in the 2013 Advancing IPF Research survey. In total, there were 143 respondents. GORD: gastrooesophageal reflux disease; PH: pulmonary hypertension; CV: cardiovascular.

treatments included pirfenidone, supplemental oxygen therapy and $N$-acetylcysteine (NAC). A proportion of patients were being treated with corticosteroids and immunosuppressants (fig. 5). In the French survey, a higher proportion of physicians reported using corticosteroids alone or in combination with immunosuppressants and/or NAC. However, it should be noted that the French survey took place before the results of studies such as the PANTHER trial were published [9] and just after pirfenidone first became available in France for the treatment of mild-to-moderate IPF.

\section{Management of comorbidities in IPF}

The majority of AIR participants reported that it was very important to screen for comorbidities and complications in IPF patients, and the majority of respondents always screened for emphysema, gastrooesophageal reflux disease, pulmonary hypertension and lung cancer (fig. 6). A large proportion of AIR respondents systematically treated gastro-oesophageal reflux disease in patients diagnosed with IPF but the majority only treated it if gastro-oesophageal reflux disease symptoms or a documented history were present. Regarding pulmonary hypertension, only a small proportion of AIR respondents (4\%) would "regularly" treat pulmonary hypertension if present in their IPF patients but $64 \%$ would treat it "occasionally" (off-label).

\section{Discussion}

The results of the 2013 AIR survey offer a number of insights into the current diagnosis and management of IPF. However, it is important to note the limitations of the survey. For example, responses to many of the questions in the AIR survey may be considered subjective. In addition, the comparison between the AIR survey and the French survey is only indicative, since the surveys were different in design and collected responses from different physician populations. The AIR survey responses may not give a comprehensive picture of the overall diagnosis and treatment of IPF as it mainly involved pulmonologists with an interest and experience in IPF, whereas many of the initial stages of IPF management are likely to be dealt with by physicians without such in-depth knowledge of IPF. Nevertheless, as mentioned, the responses to the 2013 AIR survey raise several important points.

There appears to be a delay of $\sim 1$ year in the diagnosis of IPF and, whilst this is less than conditions such as pulmonary arterial hypertension, this issue needs to be addressed since such an extensive delay is likely to have prognostic implications. A large number of AIR respondents suspected IPF through hearing velcro crackles on auscultation, which supports increasing awareness of the role of crackles with other healthcare professionals to aid earlier diagnosis [10].

When diagnosing IPF, $29 \%$ of AIR survey participants and 77\% of French survey participants performed genetic investigations if the patient had a positive family history of IPF. These figures are surprising given that the ATS/ERS/JRS/ALAT guidelines do not recommend such testing. It may be interesting to note which genetic investigations were performed at these centres; thus, it should be explored whether the disparity in testing between survey participants is due to a variation in the attitudes of physicians in different countries regarding IPF management. 
Over twice as many French survey participants carried out genetic testing compared with AIR survey participants, reflecting potential variation in the attitudes of physicians in both surveys and in the awareness of possible genetic causes of IPF. The French guidelines for IPF diagnosis and management recommend that, when a diagnosis of IPF is suspected in a patient, clinicians should "systematically" search for other causes of diffuse interstitial pneumonia in the family, including clinical signs suggestive of a genetic cause $[11,12]$. Furthermore, the guidelines propose that patients who present with IPF in a familial context should be referred to an outpatient clinic specialising in genetics to establish a pedigree and plan genetic molecular analysis $[11,12]$. Since the French guidelines (English version) were published after the AIR survey results, it is hoped their adoption into clinical practice will, in future, result in a greater level of homogeneity in practice regarding genetic testing for patients with suspected familial IPF.

Physicians responding to both the AIR survey and the French survey largely considered the ATS/ERS/JRS/ ALAT guidelines useful for the management of IPF. This was particularly reflected in the practice of involving the MDT in the diagnosis and management.

In the AIR survey, oxygen therapy, pirfenidone and NAC monotherapy were the treatments most commonly used for IPF. This raises the question of whether the treatment recommendations in the 2011 guidelines should be updated, as is currently being done on a national basis in many European countries $[13,14]$. Possible reasons for the prescription of corticosteroids or immunosuppressants may be that in clinical practice such therapies demonstrate a benefit in some patients with conditions similar to IPF, or that the patient themselves may be reluctant to stop certain medications. The majority of AIR survey respondents recognised the importance of treating comorbidities in IPF and many systematically treated asymptomatic gastro-oesophageal reflux disease.

In summary, the 2013 AIR survey results provide a snapshot of current diagnostic and management practices in Europe and, to a degree, reflect practices that have evolved since the French survey. These surveys suggest that there is still an outstanding need to accelerate the diagnosis of IPF and to continue to encourage MDT involvement in the diagnosis and management to ensure the best management outcomes for patients.

\section{Acknowledgements}

This article is based on the proceedings of the 2013 Advancing IPF Research (AIR) meeting (Nice, France), which was sponsored by InterMune International AG (Muttenz, Switzerland). Medical writing support was provided by Michael Smith (IntraMed International, Milan, Italy), which was funded by InterMune International AG.

\section{References}

Zolak JS, de Andrade JA. Idiopathic pulmonary fibrosis. Immunol Allergy Clin North Am 2012; 32: 473-485.

Raghu G, Collard HR, Egan JJ, et al. An official ATS/ERS/JRS/ALAT statement: idiopathic pulmonary fibrosis: evidence-based guidelines for diagnosis and management. Am J Respir Crit Care Med 2011; 183: 788-824.

3 Ley B, Collard HR, King TE Jr. Clinical course and prediction of survival in idiopathic pulmonary fibrosis. Am J Respir Crit Care Med 2011; 183: 431-440.

4 Wolters PJ, Collard HR, Jones KD. Pathogenesis of idiopathic pulmonary fibrosis. Annu Rev Pathol 2014; 9: 157-179.

5 Richeldi L, Collard HR, du Bois RM, et al. Mapping the future for pulmonary fibrosis: report from the 17th International Colloquium on Lung and Airway Fibrosis. Eur Respir J 2013; 42: 230-238.

6 American Thoracic Society, European Respiratory Society. American Thoracic Society/European Respiratory Society international multidisciplinary consensus classification of the idiopathic interstitial pneumonias. Am J Respir Crit Care Med 2002; 165: 277-304.

$7 \quad$ British Thoracic Society Interstitial Lung Disease Registry Project. Management Protocol. www.brit-thoracic.org. uk/document-library/audit-and-quality-improvement/lung-disease-registry/ild-registry-management-protocol(june-2012) Date last updated: July 30, 2013. Date last accessed: February 19, 2014.

8 Cottin V, Cadranel J, Crestani B, et al. Management of idiopathic pulmonary fibrosis in France: a survey of 1244 pulmonologists. Respir Med 2014; 108: 195-202.

9 Raghu G, Anstrom JA, King TE Jr, et al. Prednisone, azathioprine, and N-acetylcysteine for pulmonary fibrosis. N Engl J Med 2012; 366: 1968-1977.

10 Cottin V, Cordier JF. Velcro crackles: the key for early diagnosis of idiopathic pulmonary fibrosis? Eur Respir J 2012; 40: 519-521.

11 Cottin V, Crestani B, Valeyre D, et al. Recommandations pratiques pour le diagnostic et la prise en charge de la fibrose pulmonaire idiopathique. Elaborées par le centre national de référence et les centres de compétence pour les maladies pulmonaires rares sous l'égide de la Société de pneumologie de Langue Française [French practical guidelines for the diagnosis and management of idiopathic pulmonary fibrosis. From the National Reference and the Competence centers for rare diseases and the Société de Pneumologie de Langue Française]. Rev Mal Respir 2013; 30: 879-902.

12 Cottin V, Crestani B, Valeyre D, et al. Diagnosis and management of idiopathic pulmonary fibrosis: French practical guidelines. Eur Respir Rev 2014; 23: 193-214.

13 Behr J. Evidence-based treatment strategies in idiopathic pulmonary fibrosis. Eur Respir Rev 2013; 22: 163-168.

14 Xaubet A, Behr J, Bendstrup E, et al. Review of IPF diagnosis and management recommendations in Europe. Sarcoidosis Vasc Diff Lung Dis 2013; 30: 249-261. 